\title{
Genetic polymorphisms of ERCC1-118, XRCC1-399 and GSTP1-105 are associated with the clinical outcome of gastric cancer patients receiving oxaliplatin-based adjuvant chemotherapy
}

\author{
YONG-PING LIU ${ }^{1 *}$, YANG LING ${ }^{1 *}$, QIU-FENG QI $^{1}$, YA-PING ZHANG ${ }^{1}$, CHANG-SONG ZHANG ${ }^{1}$, \\ CHANG-TAI ZHU ${ }^{1}$, MEI-HUA WANG ${ }^{2}$ and YAO-DONG PAN ${ }^{3}$ \\ ${ }^{1}$ Clinical Oncology Laboratory; Departments of ${ }^{2}$ Pathology and ${ }^{3}$ Gastroenterological Surgery, \\ Changzhou Tumor Hospital Affiliated to Suzhou University, Changzhou, Jiangsu 213002, P.R. China
}

Received November 29, 2012; Accepted April 16, 2013

DOI: $10.3892 / \mathrm{mmr} .2013 .1435$

\begin{abstract}
The aim of the present study was to determine whether specific molecular parameters may serve as predictors of treatment outcomes and toxicity of oxaliplatin (OXA)-based chemotherapy, which is used as an adjuvant treatment in resected gastric cancer. All gastric cancer patients examined in the study received an OXA/5-fluorouracil chemotherapeutic regimen. Genetic polymorphisms of certain platinum-related genes were determined by the TaqMan $5^{\prime}$ nuclease assay and direct sequencing. Relapse-free survival (RFS), overall survival (OS) and toxicity were evaluated according to each genotype. Following adjustment for the most relevant clinical variables, excision repair cross-complimentary group 1 (ERCC1)-118 and X-ray repair cross-complementing protein 1 (XRCC1-399) demonstrated significant predictive value for RFS and OS. We also demonstrated that carrying at least one variant XRCC1 Arg399Gln or glutathione S-transferase $\pi 1$ (GSTP1) Ile105Val allele significantly increased the risk of any grade 3 or 4 hematological toxicity. In particular, carrying at least one variant GSTP1 Ile105Val allele was also significantly correlated with an increased risk of grade 3 or 4 gastrointestinal toxicity and neurotoxicity. Our data suggested that gastric cancer patients harboring ERCC1-118 C/C and XRCC1-399 A/G or A/A genotypes may benefit from receiving OXA-based adjuvant chemotherapy, and carrying at least one
\end{abstract}

Correspondence to: Dr Yong-Ping Liu, Clinical Oncology Laboratory, Changzhou Tumor Hospital Affiliated to Suzhou University, North Huaide Road No.1, Changzhou, Jiangsu 213002, P.R. China

E-mail: liuyongping026@yahoo.com.cn

*Contributed equally

Key words: genetic polymorphisms, gastric cancer, oxaliplatin, treatment outcome, toxicity variant XRCC1 Arg399Gln or GSTP1 Ile105Val allele may contribute to the occurrence of adverse drug effects associated with OXA-based chemotherapy.

\section{Introduction}

Gastric cancer is the second most common cause of cancerrelated mortality in Asia and worldwide $(1,2)$. Surgery remains the mainstay of curative treatment. However, following radical surgery, the majority of gastric cancer patients develop local or distant recurrence (3). Efforts to improve these poor outcomes have focused on developing effective postoperative systemic and regional adjuvant therapies. Several meta-analyses of postoperative adjuvant trials have demonstrated a significant benefit for chemotherapy-treated patients (4). However, these therapies are often limited by varying degrees of survival benefits and debilitating toxicities. As a result, pharmacogenetics, the study of specific genetic or molecular signatures that may be predictive of treatment outcomes, has gained considerable interest.

Oxaliplatin (OXA) is a third-generation diaminocyclohexane platinum compound that inhibits DNA replication by forming adducts between two adjacent guanines or an adjacent guanine and adenine. The adducts formed by OXA appear to be more effective at inhibiting DNA synthesis compared with cisplatin adducts (5). Numerous studies have confirmed the activity and tolerability of the combination of OXA and 5-fluorouracil (5-FU) modulated with leucovorin (LV) administered to patients with gastric cancer (6-9). However, resistance to OXA remains a major obstacle to further improvement of the response rate. DNA repair capacity is considered to be a crucial molecular pathway implicated in the resistance to platinum-based chemotherapy (10). Nucleotide excision repair (NER) is the primary DNA repair mechanism for the removal of bulky, helix-distorting DNA adducts, including those generated by platinum-based chemotherapy $(11,12)$, while the base excision repair (BER) system mainly repairs the small lesions around the damaged bases or single-strand breaks (SSBs) $(13,14)$. Single nucleotide polymorphisms in DNA 
Table I. Genetic markers evaluated in this study.

\begin{tabular}{lllrrr}
\hline Polymorphism substitution & dbSNP & NCBI Ref Seq & Exon & Genotype & Amino acid \\
\hline ERCC Asn118Asn & rs11615 & NM_001983 & 4 & C/T & Asn/118 \\
XRCC Arg399Gln & rs25487 & NM_006297 & 10 & G/A & Gln/399 \\
XPD Lys751Gln & rs13181 & NM_00400 & 23 & A/G & Val/751 \\
GSTP1 Ile105Val & rs1695 & NM_000852 & 5 & A/G & Val/105 \\
\hline
\end{tabular}

ERCC, excision repair cross-complimentary group; XRCC, X-ray repair cross-complementing protein; XPD, xeroderma pigmentosum group D; GSTP1, glutathione S-transferase $\pi$ 1; dbSNP, Single Nucleotide Polymorphism Database; NCBI, National Center for Biotechnology Information.

repair genes may be correlated with DNA-repair capacity and may affect the response to platinum-based chemotherapy (10).

The multifunctional detoxifying glutathione S-transferase (GST) enzymes have been specifically implicated in the metabolism of platinum drugs (15) and the polymorphisms of the GST family may affect platinum efficacy by lowering the intracellular concentrations of drugs.

Numerous genomic polymorphisms in genes encoding DNA-repair enzymes and detoxification enzymes have been shown to be correlated with the response to platinum-based chemotherapy. Among which, the four most commonly studied gene polymorphisms correlated with the sensitivity of cancer cells to platinum-based chemotherapy are excision repair cross-complimentary group 1 (ERCC1) Asp118Asp, X-ray repair cross-complementing protein 1 (XRCC1) Arg399Gln, xeroderma pigmentosum group D (XPD) Lys751Gln and glutathione S-transferase $\pi 1$ (GSTP1) Ile105Val. However, the association between these gene polymorphisms and the clinical end points are controversial. In addition, little is known with regard to the correlation between these putative markers and survival or toxicity in gastric cancer patients receiving OXA-based adjuvant chemotherapy. The aim of the present study was to assess the correlation between the four SNPs and survival/toxicity in a series of consecutive resected gastric cancer patients treated with OXA/5-FU adjuvant chemotherapy.

\section{Materials and methods}

Patients and treatment protocols. Blood samples were obtained from 126 patients with stage Ib-III disease, who were recruited during the period between October 2004 and March 2007 and underwent surgery at the Department of Gastroenterological Surgery, Changzhou Tumor Hospital (Jiangsu, China). The patients comprised of 90 males and 36 females (range, 30-78 years of age; median age, 57 years). None of the patients had previously received chemotherapy. This study was approved by the local ethics committee of The Changzhou Tumor Hospital (Changzhou, China) and written informed consent was obtained from all patients. Following surgery, all patients received $\geq 8$ cycles of $85 \mathrm{mg} / \mathrm{m}^{2}$ OXA plus $20 \mathrm{mg}$ / $\mathrm{m}^{2} \mathrm{LV}$ on the first day of treatment, followed by 5 -FU via a $400 \mathrm{mg} / \mathrm{m}^{2}$ bolus, and a 22-h continuous infusion of $600 \mathrm{mg} / \mathrm{m}^{2}$ 5-FU on days 1-2 at 2-week intervals. Side effects were graded according to the United States National Cancer Institute (NCI)
Common Toxicity Criteria, version 2.0 (16). OXA dose reductions of $25 \%$ were performed in cases of grade 4 hematological toxicity, febrile neutropenia and persistent paresthesias (P14 d) or painful, temporary paresthesias (7-14 d). If hematological and non-hematological toxicities had not recovered prior to the next treatment cycle, the OXA dose was delayed for a maximum of 2 weeks. If these toxicities had not recovered by that time, patients were removed from the study. All patients received a cumulative OXA dose of $\geq 500 \mathrm{mg} / \mathrm{m}^{2}$. Prophylactic use of hematological growth factors was not permitted.

Genotyping. DNA extractions from peripheral blood samples were performed using the QiaAmp kit (Qiagen, Valencia, CA, USA). SNPs in ERCC1 Asp118Asp, XPD Lys751Gln, XRCC1 Arg399Gln and GSTP1 Ile105Val (Table I) were assessed by a 5' nuclease allelic discrimination assay (Applied Biosystems, Foster City, CA, USA) using a fluorescent temperature cycler (icycler iQ Multicolor Real-time PCR Detection system; Bio-Rad Laboratories, Inc., Hercules, CA, USA). Each reaction contained the template DNA and a final concentration of 1X TaqMan PCR Master Mix (Applied Biosystems, Foster City, CA, USA), $300 \mathrm{nM}$ of each primer, $100 \mathrm{nM}$ of wild-type probe (Applied Biosystems) and $100 \mathrm{nM}$ of variant probe (Applied Biosystems). The PCR conditions were $50^{\circ} \mathrm{C}$ for $2 \mathrm{~min}$ and $95^{\circ} \mathrm{C}$ for $15 \mathrm{~min}$, followed by 45 cycles at $95^{\circ} \mathrm{C}$ for $15 \mathrm{sec}$ and $60^{\circ} \mathrm{C}$ for $1 \mathrm{~min}$. For each SNP, sequencing was performed using an ABI 3730 Genetic Analyzer (Applied Biosystems). Those with concordant results from the two analyses were included in the final data analysis.

Follow-up. Interim history, physical examination, hematological studies, carcinoembryonic antigen levels and whole-body computed tomography were performed every 2 months in the first year and every 6 months thereafter. Patients underwent upper endoscopy 3 months following surgery and every 6 months thereafter. The recurrences or metastases of gastric carcinoma were confirmed by cytology and biopsy, surgery or whole-body computed tomography. The Union for International Cancer Control (UICC) staging system (7th version) was used for the classification of each case. The study was conducted in a blind fashion, so that the patient outcome was unknown to the investigators performing the molecular analysis. Relapse-free survival (RFS) was the time from study entry until disease recurrence, mortality or the day of the last follow-up visit (whichever occurred first). Overall survival (OS) was the time 
Table II. Factors correlated with survival in patients receiving surgery followed by oxaliplatin-based adjuvant chemotherapy.

\begin{tabular}{|c|c|c|c|c|c|}
\hline Characteristic & $\mathrm{n}$ & M-RFS (months) & P-value & MST (months) & P-value \\
\hline Gender & & & 0.177 & & 0.513 \\
\hline Male & 90 & 16 & & 27 & \\
\hline Female & 36 & 12 & & 24 & \\
\hline Age (years) & & & 0.553 & & 0.912 \\
\hline$\leq 57$ & 54 & 13 & & 24 & \\
\hline$>57$ & 72 & 15 & & 26 & \\
\hline ECGO PS & & & $<0.001$ & & $<0.001$ \\
\hline 0 or 1 & 90 & 39 & & 45 & \\
\hline$>2$ & 36 & 5 & & 15 & \\
\hline Tumor differentiation & & & 0.147 & & 0.112 \\
\hline Well differentiated & 28 & 21 & & & \\
\hline Moderately differentiated & 80 & 16 & & 27 & \\
\hline Undifferentiated & 18 & 5 & & 14 & \\
\hline Tumor location & & & 0.018 & & 0.020 \\
\hline Proximal stomach & 40 & 12 & & 18 & \\
\hline Stomach body & 31 & & & & \\
\hline Distal stomach & 55 & 13 & & 24 & \\
\hline Staging & & & $<0.001$ & & $<0.001$ \\
\hline $\mathrm{Ib}+\mathrm{II}$ & 19 & & & & \\
\hline III & 107 & 12 & & 21 & \\
\hline ERCC1-118 & & & $<0.001$ & & $<0.001$ \\
\hline $\mathrm{C} / \mathrm{C}$ & 81 & 45 & & & \\
\hline $\mathrm{C} / \mathrm{T}+\mathrm{T} / \mathrm{T}$ & 45 & 5 & & 15 & \\
\hline XRCC1-399 & & & 0.001 & & $<0.001$ \\
\hline $\mathrm{G} / \mathrm{G}$ & 71 & 8 & & 18 & \\
\hline $\mathrm{A} / \mathrm{G}+\mathrm{A} / \mathrm{A}$ & 55 & 47 & & & \\
\hline XPD-751 & & & 0.639 & & 0.769 \\
\hline $\mathrm{A} / \mathrm{A}$ & 107 & 15 & & 26 & \\
\hline $\mathrm{A} / \mathrm{G}$ & 19 & 12 & & 24 & \\
\hline GSTP1-105 & & & 0.033 & & 0.019 \\
\hline $\mathrm{A} / \mathrm{A}$ & 86 & 12 & & 21 & \\
\hline $\mathrm{A} / \mathrm{G}+\mathrm{G} / \mathrm{G}$ & 40 & 47 & & & \\
\hline CEA (ng/ml) & & & $<0.001$ & & $<0.001$ \\
\hline$\leq 5$ & 79 & 47 & & & \\
\hline$>5$ & 47 & 6 & & 16 & \\
\hline
\end{tabular}

M-RFS, median replase-free survival time; MST, median survival time; ECOG PS, Eastern Cooperative Oncology Group performance status; ERCC1, excision repair cross-complimentary group 1; XRCC1, X-ray repair cross-complementing protein 1; XPD, xeroderma pigmentosum group D; GSTP1, glutathione S-transferase $\pi$; CEA, carcinoembryonic antigen.

from study entry until the date of death, regardless of the cause, or the most recent documented follow-up.

Statistical analysis. Statistical significance was based on a two-sided significance level of 0.05. All analyses were performed with SPSS (version 13.0; SPSS Inc., Chicago, IL, USA). The correlation between different genotypes and the clinical variables or toxicity to chemotherapy were tested by the $\chi^{2}$ test or Fisher's exact test (two-sided), as appropriate. The Kaplan-Meier survival method was used to estimate the survival curves, and the log-rank test was used to analyze univariate distributions for RFS and OS. The prognostic significance of the different gene SNPs following adjustment for other prognostic factors was assessed using the Cox proportional hazards regression model.

\section{Results}

Patients. A total of 126 gastric cancer patients comprised of 90 males and 36 females (range, 30-78 years of age; median 
Table III. Hazard ratios for relapse-free survival and overall survival.

\begin{tabular}{|c|c|c|c|c|c|c|}
\hline \multirow[b]{2}{*}{ Prognostic factor } & \multicolumn{3}{|c|}{ RFS } & \multicolumn{3}{|c|}{ OS } \\
\hline & HR & $95 \% \mathrm{CI}$ & P-value & $\mathrm{HR}$ & $95 \% \mathrm{CI}$ & P-value \\
\hline ERCC1-118 & & & 0.001 & & & 0.001 \\
\hline $\mathrm{C} / \mathrm{C}$ & 1 & & & 1 & & \\
\hline $\mathrm{C} / \mathrm{T}+\mathrm{T} / \mathrm{T}$ & 2.220 & $1.392-3.540$ & & 2.262 & $1.369-3.738$ & \\
\hline XRCC1-399 & & & 0.008 & & & 0.020 \\
\hline $\mathrm{G} / \mathrm{G}$ & 1 & & & 1 & & \\
\hline $\mathrm{A} / \mathrm{G}+\mathrm{A} / \mathrm{A}$ & 0.499 & $0.298-0.836$ & & 0.508 & $0.288-0.898$ & \\
\hline Staging & & & 0.012 & & & 0.027 \\
\hline $\mathrm{Ib}+\mathrm{II}$ & 1 & & & 1 & & \\
\hline III & 13.142 & $1.782-96.919$ & & 9.648 & $1.297-71.781$ & \\
\hline CEA (ng/ml) & & & 0.017 & & & 0.005 \\
\hline$\leq 5$ & 1 & & & 1 & & \\
\hline$>5$ & 1.844 & $1.114-3.053$ & & 2.213 & $1.279-3.829$ & \\
\hline ECOG PS & & & 0.006 & & & 0.018 \\
\hline 0 or 1 & & & & & & \\
\hline 2 & 1.994 & $1.221-3.254$ & & 1.855 & $1.111-3.097$ & \\
\hline
\end{tabular}

age, 57 years). Of the total number of patients, $15.08 \%$ had stage Ib and stage II disease, and $84.92 \%$ had stage III disease at the time of diagnosis. The Eastern Cooperative Oncology Group performance status (ECOG PS) was 0-1 in 90 patients and 2 in 36 patients at the time of accepting chemotherapy. Detailed demographic and disease characteristics are listed in Table II. The median RFS time (M-RFS) was 12 months (range, 2-56 months), and the median survival time (MST) was 21 months (range, 5-56 months). The patient characteristics and their outcomes were unknown to the investigators performing the genetic analysis. The genotyping results were disclosed to the clinical investigators following data analysis.

Genotype frequencies of polymorphisms of ERCC1, XRCC1, $X P D$ and GSTP1. The results of the genotyping of ERCC1-118, XRCC1-399, XPD-751 and GSTP1-105 were available for all 126 patients. The wild genotype (C/C) of ERCC1 Asp118Asp was observed in 81 patients $(64.29 \%)$, while the heterozygous variant $(\mathrm{C} / \mathrm{T})$ was present in 36 patients $(28.57 \%)$ and the homozygous variant (T/T) was present in 9 patients $(7.14 \%)$. The wild genotype (G/G) of XRCC1 Arg399Gln was observed in 71 patients $(56.35 \%)$ and the heterozygous variant $(\mathrm{G} / \mathrm{A})$ in 33 patients $(26.19 \%)$, whereas the homozygous variant (A/A) was present in 6 patients (4.76\%). The wild type (A/A) of GSTP1 Ile105Val was present in 86 patients $(68.25 \%)$, the heterozygous variant $(\mathrm{A} / \mathrm{G})$ was observed in 35 patients $(27.78 \%)$, and the homozygous variant $(\mathrm{G} / \mathrm{G})$ was present in 5 patients $(3.97 \%)$. The wild genotype (A/A) of XPD Lys $751 \mathrm{Gln}$ was observed in 107 patients $(84.92 \%)$, while the heterozygous variant $(\mathrm{A} / \mathrm{C})$ was present in 19 patients $(15.08 \%)$, and the homozygous variant of codon 751 in the XPD gene was not observed in any patient. Genotype frequencies for ERCC1, XRCC1, XPD and GSTP1 polymorphisms were demonstrated to be in Hardy-Weinberg equilibrium. No significant correlations were observed between any of these polymorphisms and age, gender, ECOG status, initial tumor stage and grade.

Correlation between polymorphisms and survival. With regard to RFS, the three variable ERCC1 Asp118Asp, XRCC1 Arg399Gln and GSTP1 Ile105Val SNPs demonstrated a predictive value. For ERCC1 Asp118Asp, the M-RFS was 5 months for TT and C/T cases, and 45 months for $\mathrm{C} / \mathrm{C}$ patients ( $\mathrm{P}<0.001$; Fig. 1A). For XRCC1 Arg399Gln, the M-RFS was 47 months for AA and A/G cases, and 8 months for $\mathrm{G} / \mathrm{G}$ patients $(\mathrm{P}=0.001)$. Additionally, for GSTP1 Ile105Val, the M-RFS was 47 months for G/G and A/G cases, and 12 months for AA patients $(\mathrm{P}=0.033)$. Both ERCC1 Asp118Asp and XRCC1 Arg399Gln remained significant in the multivariate analysis. For ERCC1 Asp118Asp, TT and C/T patients had a 2.22-fold increased risk of recurrence compared with $\mathrm{C} / \mathrm{C}$ patients (RR, 1.392-3.540; $\mathrm{P}=0.001$ ). In addition, for $\mathrm{XRCC1}$ Arg399Gln, compared with the G/G cases, the patients with heterozygous and homozygous polymorphic variants (A/G and $\mathrm{A} / \mathrm{A}$ ) had a decreased risk of recurrence by 0.499 -fold (RR, 0.298-0.836; $\mathrm{P}=0.008$ ).

As for OS, the ERCC1 Asp118Asp, XRCC1 Arg399Gln and GSTP1 Ile105Val SNPs also retained their significant predictive value. For ERCC1-118, the median OS was 15 months for $\mathrm{T} / \mathrm{T}$ and $\mathrm{C} / \mathrm{T}$, and undefined for $\mathrm{C} / \mathrm{C}$ cases $(\mathrm{P}<0.001$; Fig. $2 \mathrm{~A}$ ). For the XRCC1 Arg399Gln, the median OS was 18 months for $\mathrm{G} / \mathrm{G}$ patients and undefined for $\mathrm{AA}$ and $\mathrm{A} / \mathrm{G}$ patients $(\mathrm{P}<0.001$; Fig. 2B). Additionally, for GSTP1 Ile105Val, the median OS 
A

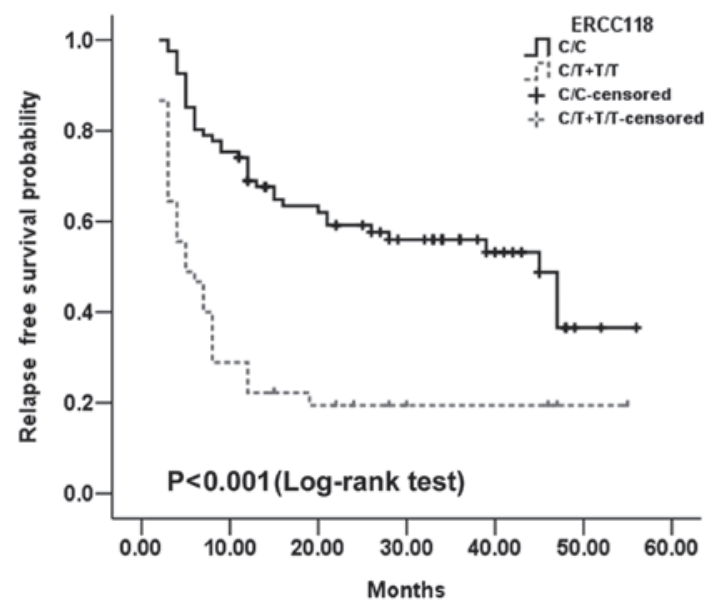

C

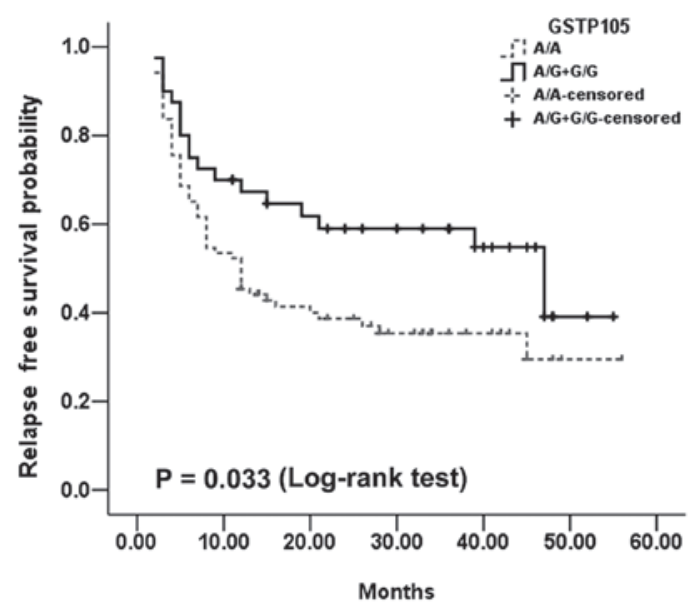

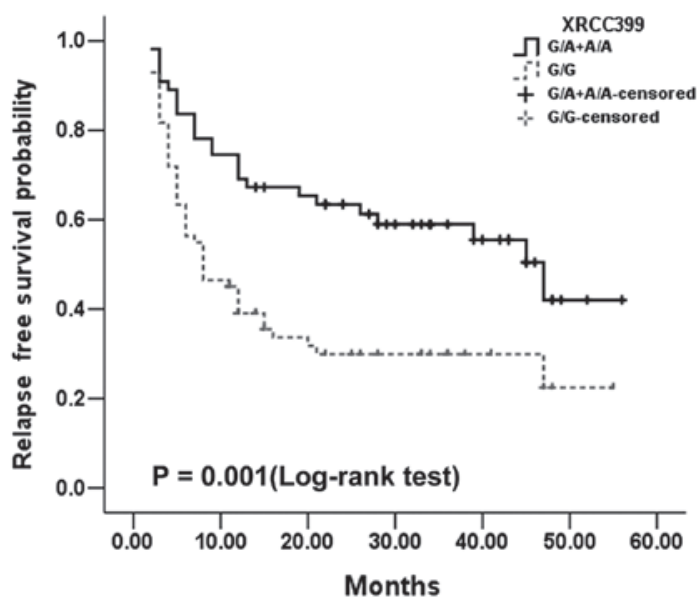

D

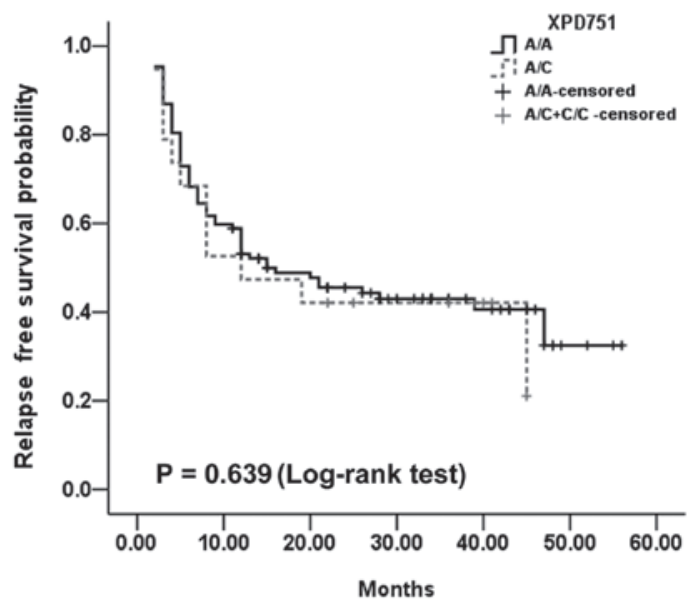

Figure 1. Relapse-free survival according to the genotype of (A) ERCC1-118; (B) XRCC1-399; (C) GSTP1-105 and (D) XPD-751. ERCC1, excision repair cross-complimentary group 1; XRCC1, X-ray repair cross-complementing protein 1; GSTP1, glutathione S-transferase $\pi$ 1; XPD, xeroderma pigmentosum group D.

was not defined for $\mathrm{G} / \mathrm{G}$ and $\mathrm{A} / \mathrm{G}$ cases, and 21 months for AA patients ( $\mathrm{P}=0.019$; Fig. 2D). Both ERCC1 Asp118Asp and XRCC1 Arg399Gln remained significant in the multivariate Cox survival analysis $[\mathrm{P}=0.001 ; \mathrm{HR}=2.262 ; 95 \%$ confidence interval $(\mathrm{CI}), 1.369-3.738$; and $\mathrm{P}=0.02 ; \mathrm{HR}=0.508 ; 95 \% \mathrm{CI}$, 0.288-03.898, respectively; Table III].

Other factors that were significantly correlated with RFS and OS in the univariate analysis using the Kaplan-Meier survival curves and the log-rank test were ECOG PS, tumor location, tumor stage and the levels of serum carcinoembryonic antigen (Table II). Gender, age and tumor differentiation were not significant prognostic factors for RFS and OS. ECOG PS, stage and serum carcinoembryonic antigen remained significant prognostic factors correlated with RFS and OS in the Cox proportional hazards regression model multivariate analysis (Table III).

Correlation between polymorphisms and toxicity. We analyzed whether the previously mentioned four gene SNPs were correlated with the severe $\mathrm{OX} / 5-\mathrm{FU}$ regimen-related toxicities in all 126 patients. There were no significant correlations between the ERCC1-118 and XPD751 polymorphisms with grade 3 or 4 toxicity. However, carrying at least one variant XRCC1
Arg399Gln and GSTP1 Ile105Val allele was significantly correlated with grade 3 or 4 hematological toxicity $(\mathrm{P}=0.029$ and $\mathrm{P}<0.001$, respectively). In particular, carrying at least one variant GSTP1 Ile105Val allele also remained significantly associated with grade 3 or 4 gastrointestinal toxicity and neurotoxicity $(\mathrm{P}=0.002$ and $\mathrm{P}=0.018$, respectively).

\section{Discussion}

The ability of cancer cells to recognize and repair DNA damage and to enhance detoxification by the GST pathway may contribute to tumor resistance to platinum-based chemotherapy $(17,18)$. In the present study, we selected four putative molecular parameters to determine whether these markers were partly responsible for sensitivity and toxicity to OXA-based chemotherapy used as adjuvant treatment in gastric cancer. Our findings supported the hypothesis that pharmacogenetic profiling may be useful for predicting the prognosis of survival and the toxicity associated with OXA-based adjuvant chemotherapy in gastric cancer patients. The univariate analysis revealed that ERCC1-118, XRCC1-399 and GSTP1-105 polymorphisms were significantly correlated with RFS $(\mathrm{P}<0.001, \mathrm{P}=0.001$ and $\mathrm{P}=0.033$, respectively) and 
A

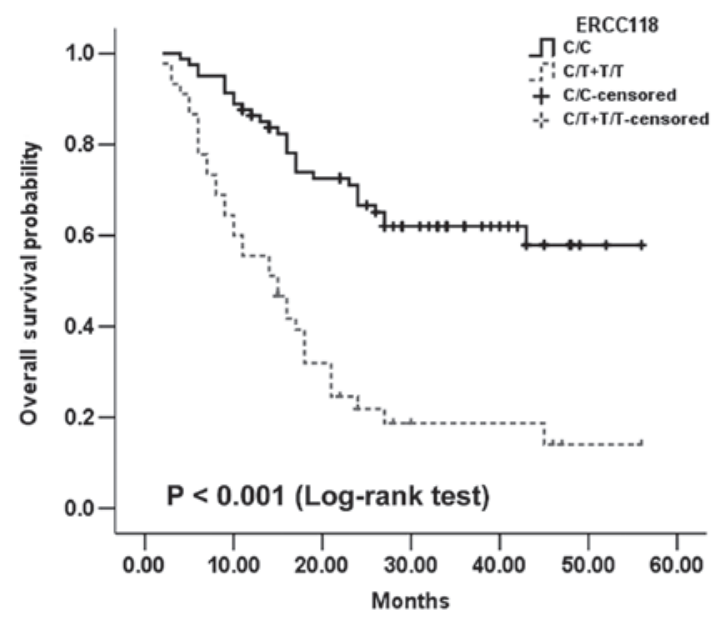

C

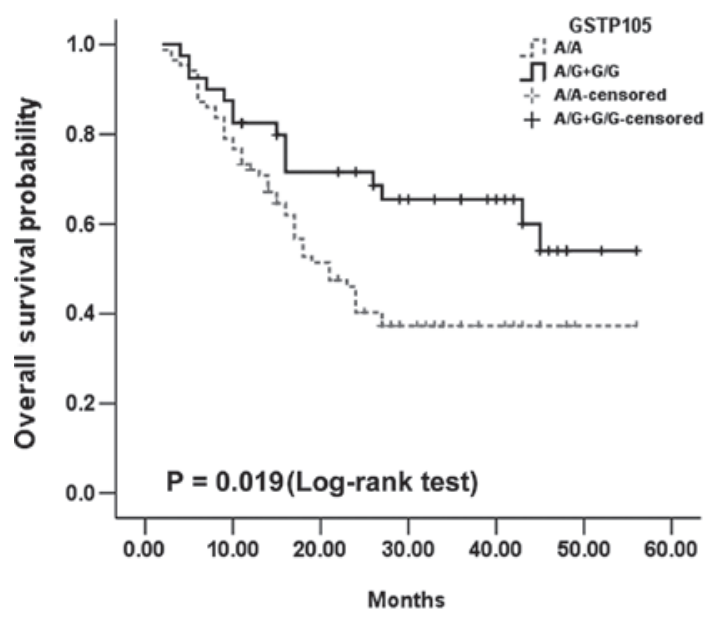

B

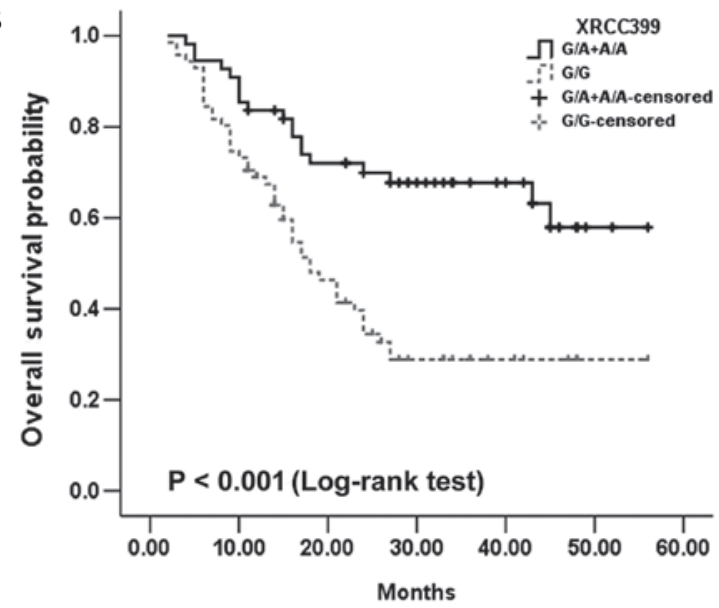

D

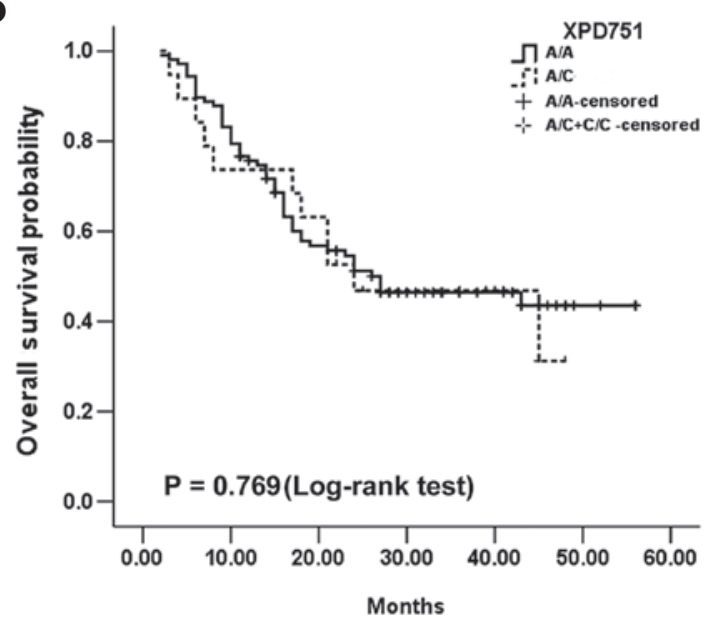

Figure 2. Overall survival according to the genotype of (A) ERCC1-118; (B) XRCC1-399; (C) GSTP1-105 and (D) XPD-751. ERCC1, excision repair cross-complimentary group 1; XRCC1, X-ray repair cross-complementing protein 1; GSTP1, glutathione S-transferase $\pi$ 1; XPD, xeroderma pigmentosum group D.

OS $(\mathrm{P}<0.001, \mathrm{P}<0.001$ and $\mathrm{P}=0.019$, respectively). The Cox proportional hazards regression model multivariate analysis suggested that ERCC1-118 and XRCC1-399 polymorphisms also retained significant predictive value for $\mathrm{RFS}(\mathrm{P}=0.001$ and $\mathrm{P}=0.008$, respectively) and $\mathrm{OS}(\mathrm{P}=0.001$ and $\mathrm{P}=0.02$, respectively). However, we also demonstrated that carrying at least one variant XRCC1 Arg399Gln or GSTP1 Ile105Val allele was significantly correlated with grade 3 or 4 hematological toxicity $(\mathrm{P}=0.029$ and $\mathrm{P}<0.001$, respectively). In particular, carrying at least one variant GSTP1 Ile105Val allele remained significantly correlated with grade 3 or 4 gastrointestinal toxicity and neurotoxicity $(\mathrm{P}=0.002$ and $\mathrm{P}=0.018$, respectively).

NER is the main mechanism in mammalian cells for the removal of bulky, helix-distorting DNA adducts produced by platinum agents (11). ERCC1 is an important DNA repair gene with critical roles in the NER pathway, which is the most important system for repairing a wide variety of structural DNA lesions, including bulky DNA adducts (19). Several studies have demonstrated that patients, including those with gastric cancer, with low ERCC1 expression were more sensitive to platinum-based chemotherapy (20-23). In vitro experiments have also demonstrated that cells with low ERCC1 expression were more sensitive to platinum derivatives or alkylating agents (24). Functional variants of the gene may alter the levels of ERCC1 gene expression. Chang et al (25) revealed that higher ERCC1 protein expression levels were correlated with the variant ERCC1-118 T allele, which may lead to resistance to platinum derivatives. This may explain the lower RFS and OS times observed in the individuals in our study of FOLFOX4 adjuvant chemotherapy (Tables II and III; Figs. 1 and 2) and other studies where patients have been treated with platinum-based chemotherapy (26-28). However, Yu et al obtained contrary results in studies of ovarian cell lines, where the ERCC1 codon 118 C-T substitution was associated with reduced levels of ERCC1 mRNA and protein expression (29). The contrasting results from different clinical studies of the ERCC-1 polymorphism (30) may be due to insufficient sample sizes; with more definitive results likely to be achieved through a large sample, multicenter prospective studies should be conducted in the future. We did not observe a correlation between the ERCC-1 polymorphism and overall toxicity, which suggests that ERCC1 may be not involved in adverse reactions to FOLFOX4 treatment, and is consistent with the results of a previous study (28).

XPD is another important component of NER. The majority of studies have demonstrated that variance in the DNA sequence of the ERCC2/XPD gene 751 was correlated with impaired DNA repair activity $(31,32)$, while one study 
demonstrated the opposite results (33). A significant correlation has also been observed between homozygosity for the wild-type XPD 751 allele (Lys/Lys) and an improved response to FU-OXA in metastatic colorectal cancer (34). By contrast, another study in patients with stage III and IV gastric cancer treated with surgery following radiation therapy plus FU/LV-based chemotherapy obtained the opposite result; patients with the wild-type XPD-751 allele (Lys/Lys) were more likely to have relapse compared with those with Lys/Gln and Gln/Gln genotypes (35). In the present study, we did not observe a significant correlation between XPD-751 polymorphism and clinical outcome in gastric cancer patients treated with FOLFOX4 adjuvant chemotherapy, which is consistent with a previous study in non-small cell lung cancer (36). At the same time, no $\mathrm{C} / \mathrm{C}$ genotype was detected in the present study, which is consistent with the findings of studies examining the Chinese population $(37,38)$. These results suggest that the polymorphisms may differ according to ethnicity.

Base excision repair, another critical DNA repair mechanism, is also important in the response to platinum-based therapy. XRCC1 is a key player in the BER pathway. In vitro assays have demonstrated that reduced DNA repair capacity is associated with the XRCC1-399 polymorphism, and the rate of irradiation-specific DNA repair decreased with an increasing number of variant XRCC1 Arg399Gln alleles $(39,40)$. Several studies have demonstrated a correlation between XRCC1-399 G-A substitution with improved outcomes in patients with solid tumors treated with platinum-based chemotherapy (41-43). Consistent with the above results, in the present study, patients carrying at least one variant XRCC1-399A allele had a better prognosis, which may have been correlated with enhanced sensitivity to OXA-based chemotherapy. We also identified that variance in the XRCC1 Arg399Gln allele was significantly correlated with grade 3 or 4 hematological toxicity, which may have been due to the less proficient DNA repair activity. However, opposite results demonstrated an improved survival for patients with the XRCC1-399 $\mathrm{G}$ allele receiving platinum-based chemotherapy for colorectal, lung, esophageal, gastric and cervical carcinoma $(26,30,38,44-50)$. Several studies have also demonstrated that no statistically significant correlation was identified between the XRCC1 codon 399 polymorphism and survival or toxicities correlated with platinum-based chemotherapy. The aforementioned conflicting results may be due to different study populations, chemotherapy regimens and genotyping methods.

GSTs participate in the detoxification of a variety of chemotherapeutics, including platinum. The GSTP1-105A allele may be correlated with lower GSTP1 enzyme activity in the tumor tissue (51). In the present study, we verified that patients with GSTP1-105A allele variants not only exhibited longer relapse-free $(\mathrm{P}<0.01)$ and overall $(\mathrm{P}<0.01)$ survival times; however, also had a higher incidence of grade $3 / 4$ cumulative neuropathy, gastrointestinal toxicity and hematological toxicity following different cycles of treatment, which is partly in accordance with the results of Stoehlmacher et al (52). The aforementioned results may be correlated with the reduced metabolism and slower removal of chemotherapeutic agents, which yields a longer RFS and OS; however, leads to toxicity of platinum-based chemotherapy.
Although our findings supported the theory that ERCC1 Asp118Asp, XRCC1 Arg399GIn and GSTP1 Ile105Val polymorphisms may be useful for predicting the prognosis of survival and the toxicity associated with OXA-based adjuvant chemotherapy in gastric cancer patients, the limitations of our study must be acknowledged. These include insufficient sample sizes and a single unit population. Therefore, larger sample sizes, multicenter prospective studies and even basic functional studies are required to confirm the results and identify the biological basis of these findings.

In conclusion, the results of the present study indicate that pharmacogenetic profiling may be useful for predicting the prognosis of survival and the toxicity associated with the OXA-based adjuvant chemotherapy in gastric cancer patients.

\section{Acknowledgements}

The authors would like to thank the personnel at the Changzhou Tumor Hospital (Changzhou, China) in our study. The study was partly supported by the Science and Technology Planning Project of Changzhou, Jiangsu (CS20092025); the Research of Health Department in Jiangsu (Z201221); the Science and Technology Planning Project of Changzhou Health Bureau, Jiangsu (QN201106 and ZD201203); the 333 Talents Training Project of Jiangsu Province; the Key Medical Innovation Talents Training Project of Changzhou, Jiangsu and the Project of Jiangsu Province Sanitation Innovation Team.

\section{References}

1. Kamangar F, Dores GM and Anderson WF: Patterns of cancer incidence, mortality, and prevalence across five continents: defining priorities to reduce cancer disparities in different geographic regions of the world. J Clin Oncol 24: 2137-2150, 2006.

2. Leung WK, Wu MS, Kakugawa Y, et al: Screening for gastric cancer in Asia: current evidence and practice. Lancet Oncol 9: 279-287, 2008

3. Macdonald JS: Treatment of localized gastric cancer. Semin Oncol 31: 566-573, 2004.

4. Carrato A, Gallego-Plazas J and Guillen-Ponce C: Adjuvant therapy of resected gastric cancer is necessary. Semin Oncol 32: S105-S108, 2005.

5. Mamenta EL, Poma EE, Kaufmann WK, Delmastro DA, Grady HL and Chaney SG: Enhanced replicative bypass of platinum-DNA adducts in cisplatin-resistant human ovarian carcinoma cell lines. Cancer Res 54: 3500-3505, 1994.

6. Louvet C, André T, Tigaud JM, et al: Phase II study of oxaliplatin, fluorouracil, and folinic acid in locally advanced or metastatic gastric cancer patients. J Clin Oncol 20: 4543-4548, 2002.

7. De Vita F, Orditura M, Matano E, et al: A phase II study of biweekly oxaliplatin plus infusional 5-fluorouracil and folinic acid (FOLFOX-4) as first-line treatment of advanced gastric cancer patients. Br J Cancer 92: 1644-1649, 2005.

8. Al-Batran SE, Atmaca A, Hegewisch-Becker S, et al: Phase II trial of biweekly infusional fluorouracil, folinic acid, and oxaliplatin in patients with advanced gastric cancer. J Clin Oncol 22: 658-663, 2004.

9. Cavanna L, Artioli F, Codignola C, et al: Oxaliplatin in combination with 5-fluorouracil (5-FU) and leucovorin (LV) in patients with metastatic gastric cancer (MGC). Am J Clin Oncol 29: 371-375, 2006.

10. Wei Q, Frazier ML and Levin B: DNA repair: a double-edged sword. J Natl Cancer Inst 92: 440-441, 2000.

11. Reardon JT, Vaisman A, Chaney SG and Sancar A: Efficient nucleotide excision repair of cisplatin, oxaliplatin, and Bis-aceto-ammine-dichloro-cyclohexylamineplatinum(IV) (JM216) platinum intrastrand DNA diadducts. Cancer Res 59: 3968-3971, 1999. 
12. Reed E: Platinum-DNA adduct, nucleotide excision repair and platinum based anti-cancer chemotherapy. Cancer Treat Rev 24: 331-344, 1998.

13. Goode EL, Ulrich CM and Potter JD: Polymorphisms in DNA repair genes and associations with cancer risk. Cancer Epidemiol Biomarkers Prev 11: 1513-1530, 2002.

14. Wood RD, Mitchell M, Sgouros J and Lindahl T: Human DNA repair genes. Science 291: 1284-1289, 2001

15. Frankmoelle WP, Medina JC, Shan B, Narbut MR and Beckmann H: Glutathione S-transferase metabolism of the antineoplastic pentafluorophenylsulfonamide in tissue culture and mice. Drug Metab Dispos 28: 951-958, 2000.

16. National Cancer Institute: NCI Common Toxicity Criteria. http:// ctep.cancer.gov. Accessed October 1, 2004.

17. Salinas AE and Wong MG: Glutathione S-transferases - a review. Curr Med Chem 6: 279-309, 1999.

18. Gossage L and Madhusudan S: Cancer pharmacogenomics: role of DNA repair genetic polymorphisms in individualizing cancer therapy. Mol Diagn Ther 11: 361-380, 2007.

19. De Silva IU, McHugh PJ, Clingen PH and Hartley JA: Defining the roles of nucleotide excision repair and recombination in the repair of DNA interstrand cross-links in mammalian cells. Mol Cell Biol 20: 7980-7990, 2000.

20. Weberpals J, Garbuio K, O'Brien A, et al: The DNA repair proteins BRCA1 and ERCC1 as predictive markers in sporadic ovarian cancer. Int J Cancer 124: 806-815, 2009.

21. Denlinger CS and Cohen SJ: Progress in the development of prognostic and predictive markers for gastrointestinal malignancies. Curr Treat Options Oncol 8: 339-351, 2007.

22. Iqbal S, Stoehlmacher J and Lenz HJ: Tailored chemotherapy for colorectal cancer: a new approach to therapy. Cancer Invest 22 762-773, 2004

23. Hofler H, Langer R, Ott K and Keller G: Prediction of response to neoadjuvant chemotherapy in carcinomas of the upper gastrointestinal tract. Adv Exp Med Biol 587: 115-120, 2006.

24. Guichard S, Arnould S, Hennebelle I, Bugat R and Canal P. Combination of oxaliplatin and irinotecan on human colon cancer cell lines: activity in vitro and in vivo. Anticancer Drugs 12: 741-751, 2001.

25. Chang PM, Tzeng $\mathrm{CH}$, Chen PM, et al: ERCCl codon $118 \mathrm{C} \rightarrow \mathrm{T}$ polymorphism associated with ERCC1 expression and outcome of FOLFOX-4 treatment in Asian patients with metastatic colorectal carcinoma. Cancer Sci 100: 278-283, 2009.

26. Kalikaki A, Kanaki M, Vassalou H, et al: DNA repair gene polymorphisms predict favorable clinical outcome in advanced non-small-cell lung cancer. Clin Lung Cancer 10: 118-123, 2009.

27. Stoehlmacher J, Park DJ, Zhang W, et al: A multivariate analysis of genomic polymorphisms: prediction of clinical outcome to 5-FU/oxaliplatin combination chemotherapy in refractory colorectal cancer. Br J Cancer 91: 344-354, 2004.

28. Ruzzo A, Graziano F, Loupakis F, et al: Pharmacogenetic profiling in patients with advanced colorectal cancer treated with first-line FOLFOX-4 chemotherapy. J Clin Oncol 25: 1247-1254, 2007.

29. Yu JJ, Lee KB, Mu C, et al: Comparison of two human ovarian carcinoma cell lines (A2780/CP70 and MCAS) that are equally resistant to platinum, but differ at codon 118 of the ERCC1 gene. Int J Oncol 16: 555-560, 2000.

30. Huang MY, Huang ML, Chen MJ, et al: Multiple genetic polymorphisms in the prediction of clinical outcome of metastatic colorectal cancer patients treated with first-line FOLFOX-4 chemotherapy. Pharmacogenet Genomics 21: 18-25, 2011.

31. Spitz MR, Wu X, Wang Y, et al: Modulation of nucleotide excision repair capacity by XPD polymorphisms in lung cancer patients. Cancer Res 61: 1354-1357, 2001.

32. Wolfe KJ, Wickliffe JK, Hill CE, Paolini M, Ammenheuser MM and Abdel-Rahman SZ: Single nucleotide polymorphisms of the DNA repair gene XPD/ERCC2 alter mRNA expression. Pharmacogenet Genomics 17: 897-905, 2007.

33. Lunn RM, Helzlsouer KJ, Parshad R, et al: XPD polymorphisms: effects on DNA repair proficiency. Carcinogenesis 21: 551-555, 2000.

34. Park DJ, Stoehlmacher J, Zhang W, Tsao-Wei DD, Groshen S and Lenz HJ: A Xeroderma pigmentosum group D gene polymorphism predicts clinical outcome to platinum-based chemotherapy in patients with advanced colorectal cancer. Cancer Res 61: 8654-8658, 2001.
35. Zárate RN, Arias F, Bandres E, Cubedo E, Malumbres R and García-Foncillas J: Xeroderma pigmentosum group D 751 polymorphism as a predictive factor in resected gastric cancer treated with chemo-radiotherapy. World J Gastroenterol 12: 6032-6036, 2006.

36. Tibaldi C, Giovannetti E, Vasile E, et al: Correlation of CDA ERCC1, and XPD polymorphisms with response and survival in gemcitabine/cisplatin-treated advanced non-small cell lung cancer patients. Clin Cancer Res 14: 1797-1803, 2008.

37. Xing D, Tan W, Wei Q and Lin D: Polymorphisms of the DNA repair gene XPD and risk of lung cancer in a Chinese population. Lung Cancer 38: 123-129, 2002.

38. Liu B, Wei J, Zou Z, et al: Polymorphism of XRCC1 predicts overall survival of gastric cancer patients receiving oxaliplatin-based chemotherapy in Chinese population. Eur J Hum Genet 15: 1049-1053, 2007.

39. Vodicka P, Kumar R, Stetina R, et al: Genetic polymorphisms in DNA repair genes and possible links with DNA repair rates, chromosomal aberrations and single-strand breaks in DNA. Carcinogenesis 25: 757-763, 2004.

40. Vodicka P, Stetina R, Polakova V, et al: Association of DNA repair polymorphisms with DNA repair functional outcomes in healthy human subjects. Carcinogenesis 28: 657-664, 2007.

41. Pacetti P, Giovannetti E, Mambrini A, et al: Single nucleotide polymorphisms and clinical outcome in patients with biliary tract carcinoma treated with epirubicin, cisplatin and capecitabine. Anticancer Res 29: 1835-1840, 2009.

42. Giachino DF, Ghio P, Regazzoni S, et al: Prospective assessment of XPD Lys751Gln and XRCC1 Arg399Gln single nucleotide polymorphisms in lung cancer. Clin Cancer Res 13: 2876-2881, 2007.

43. Moreno V, Gemignani F, Landi S, et al: Polymorphisms in genes of nucleotide and base excision repair: risk and prognosis of colorectal cancer. Clin Cancer Res 12: 2101-2108, 2006.

44. Suh KW, Kim JH, Kim do Y, Kim YB, Lee C and Choi S: Which gene is a dominant predictor of response during FOLFOX chemotherapy for the treatment of metastatic colorectal cancer, the MTHFR or XRCC1 gene? Ann Surg Oncol 13: 1379-1385, 2006.

45. de las Peñas R, Sanchez-Ronco M, Alberola V, et al: Polymorphisms in DNA repair genes modulate survival in cisplatin/gemcitabine-treated non-small-cell lung cancer patients. Ann Oncol 17: 668-675, 2006.

46. Bewick MA, Conlon MS and Lafrenie RM: Polymorphisms in $\mathrm{XRCC1}$, XRCC3, and CCND1 and survival after treatment for metastatic breast cancer. J Clin Oncol 24: 5645-5651, 2006.

47. Yoon HH, Catalano PJ, Murphy KM, et al: Genetic variation in DNA-repair pathways and response to radiochemotherapy in esophageal adenocarcinoma: a retrospective cohort study of the Eastern Cooperative Oncology Group. BMC Cancer 11: 176, 2011.

48. Cheng XD, Lu WG, Ye F, Wan XY and Xie X: The association of XRCC1 gene single nucleotide polymorphisms with response to neoadjuvant chemotherapy in locally advanced cervical carcinoma. J Exp Clin Cancer Res 28: 91, 2009.

49. Huang ZH, Hua D and Du X: Polymorphisms in p53, GSTP1 and XRCC1 predict relapse and survival of gastric cancer patients treated with oxaliplatin-based adjuvant chemotherapy. Cancer Chemother Pharmacol 64: 1001-1007, 2009.

50. Goekkurt E, Al-Batran SE, Hartmann JT, et al: Pharmacogenetic analyses of a phase III trial in metastatic gastroesophageal adenocarcinoma with fluorouracil and leucovorin plus either oxaliplatin or cisplatin: a study of the arbeitsgemeinschaft internistische onkologie. J Clin Oncol 27: 2863-2873, 2009.

51. Kweekel DM, Koopman M, Antonini NF, et al: GSTP1 Ile105Val polymorphism correlates with progression-free survival in MCRC patients treated with or without irinotecan: a study of the Dutch Colorectal Cancer Group. Br J Cancer 99: 1316-1321, 2008.

52. Stoehlmacher J, Park DJ, Zhang W, et al: Association between glutathione S-transferase P1, T1, and M1 genetic polymorphism and survival of patients with metastatic colorectal cancer. J Natl Cancer Inst 94: 936-942, 2002. 